Colloque C2, suppl. au Journal de Physique II, Vol. 1, septembre 1991

\title{
PLASMAS : SOURCES OF EXCITED, DISSOCIATED AND IONIZED SPECIES. CONSEQUENCES FOR CHEMICAL VAPOR DEPOSITION (CVD) AND FOR SURFACE TREATMENT
}

\author{
A. GICQUEL ${ }^{*}$ and Y. CATHERINE* * \\ * Laboratoire d'Ingéniérie des Matériaux et des Hautes \\ Pressions, Université Paris-Nord, CNRS-UP 1311, avenue \\ Jean-Baptiste Clément, F-93430 Villetaneuse, France \\ * "Laboratoire des Plasmas et des Couches Minces. Institut des \\ Matériaux de Nantes, 2 rue de la Houssinière, F-44087 Nantes \\ Cedex, France
}

This keynote lecture is dedicated to the memory of the late Yves Catherine, who was Professor at the University of Nantes. Yves Catherine had an international reputation in the field of plasma science, in particular for his work in the deposition of amorphous silicon and diamond-like carbon.

\begin{abstract}
The use of the Plasma Enhanced Chemical Vapor Deposition techniques have increased during the last decades. PECVD attractiveness, basically due to the lowering of the substrate temperatures, has enlarged its uses because it allows an action of ions or excited species. However, the choice of the reactors is not always easy. After presenting the main domains of applications of the PECVD techniques, we describe the principal plasma reactors together with their specific characteristics. The basic phenomena involved in the flowing plasma and the attempts for their modelling are then presented. Finally, the consequences for the deposition rate and the efficiency of the surface treatment are discussed.
\end{abstract}

\section{Introduction}

It is now well known that the use of glow discharge allows activation of chemical reactions, forming the active species necessary for the deposition of thin films. Temperatures as low as 200 to $400^{\circ} \mathrm{C}$ are typical of Plasma Enhanced Chemical Vapor Deposition (PECVD) processes while 900 to $1000^{\circ} \mathrm{C}$ are more characteristic of thermally activated processes. For instance, the deposition of TiN on stainless steel can be realized in a thermal CVD apparatus, fed with $\mathrm{TiCl}_{4}$ and $\mathrm{N}_{2}$ and operating at around $950^{\circ} \mathrm{C}$, or in a PECVD reactor at $300^{\circ} \mathrm{C}[1,2]$. Consequently, an increased number of CVD techniques are now designed for working with an electrical discharge. The active species necessary for the deposition are generated in the glow discharge via electron-molecule collisional reactions and also via molecule - molecule reactions, the net result of which being the creation of a highly dissociated or ionized medium. There are mainly two types of reactors, with completly different designs and features, according to the fact that ions are desired or not for the coating growth. In the case where the ions could induce some damage on the films, the reactive neutral species, created in the plasma, such as radicals or atoms adsorb on the surface much more easily than their parent molecule. In the case where the ions participate in the deposition, they are strongly accelerated in the cathodic sheath towards the surface, and consequently increase the mobility of the adspecies and the desorption of volatile components, even at room temperature. This possibility of directing well controlled energy ions onto the surface is one of the advantages of the PECVD. This enhances both the densification of the layers in the case of amorphous or microcrystalline compounds and the adhesion because of the intermixing of the first layers with the substrate atoms.

The attractiveness of PECVD techniques, beyond the low deposition temperature, is the flexibility in using either radicals and/or atoms or ions, the possibility of successive treatments allowing multilayer deposition inside the same apparatus, and the ability of doping during the deposition stage. Moreover, the use of PECVD, because of the non equilibrium state of the plasma, is susceptible to produce gaseous species which would not form under equilibrium situations, and the formation of exotic deposits can be considered. These techniques have been used mainly in microelectronics for depositing dielectric thin films as silicon nitrides, silicon oxides and silicides and refractory metals thin films, in photovoltaic for producing amorphous silicon for solar cells, in metallurgy and microelectronics for realizing metal / metalloid compound such as titanium nitrides. More recently the formation of diamond like coatings and 
even diamond coatings for applications in optics, microelectronics and tribological fields have been demonstrated to be very efficient using PECVD techniques.

In this paper, we would like to present the different thin films already prepared with the help of the electrical discharges either at the industrial stage or at the research stage. We will present the different configurations of reactors together with their features, in particular we will discuss their specific operating for the creation of the desired active species and for the transport of these species to the surface. Then, we would like to illustrate the different approaches for modeling of both the reactive flux, including the main physical processes, and the plasma /surface interaction.

\section{I- Plasma Enhanced CVD Applications}

Recently, a large effort has been devoted to the preparation of new materials which have a number of associated properties of interest to industry. Both bulk and coatings are under competitive study, but we will only concentrate in this review article on the material surface, including deposited materials and superficial substrate treatment.

For around 10 years, $\mathrm{SiO}_{2}, \mathrm{Si}_{3} \mathrm{~N}_{4}$, thin metal films, amorphous silicon, polycrystalline silicon and epitaxial silicon coatings have received a great attention from the CVD community, and PECVD has been demonstrated to be very successful $[3,4,5,6,7]$. While silicon nitride, silicon oxide and silicon oxynitride need 700 to $900^{\circ} \mathrm{C}$ to be deposited by thermally activated CVD, PECVD allows the deposition at 250 to $350^{\circ} \mathrm{C}$. Thus, dielectric thin films can be deposited on temperature sensitive coatings such as gold or aluminium thin films [7]. The action of the glow discharge is to create the active species from the dissociation of $\mathrm{SiH}_{4}$ and $\mathrm{NH}_{3}$ or of $\mathrm{SiH}_{4}$ and $\mathrm{N}_{2}$. The stoechiometry of the nitride is strongly affected by the use of $\mathrm{NH}_{3}$ rather than $\mathrm{N}_{2}$, for a given discharge power and pressure. The transport of the species and the control of the concentration are also parameters to be controlled. The use of silicon-organic compounds such as tetraethylorthosilicate (TEOS) or hexamethyldisiloxane (HMDSO) or metallo-organic compounds $[6,8]$ allows a better control of the concentration of the silicon or the metal during the deposition of dielectric thin film, and therefore some progress in defining the uniformity and stoechiometry. For polycrystalline silicon, the action of the plasma does not only exist for dissociating the gases but also for increasing the mobility of the adatoms leading to the formation of crystallites [7]. According to the experimental conditions, the amorphous to polycrystalline transition is very sensitive to the combination of the nature of the gas, the dissociation yield (power, pressure), and the deposition temperature. Rief concludes that the hydrogen seems to play a positive role on the nucleation of microcrystallites, and that phosphine and diborane also decrease the transition temperature. Apparently, the ion flux is also playing a positive action since the transition temperature is lowered substantially. For the deposition of epitaxial silicon, PECVD has been used to lower the temperature to $650^{\circ} \mathrm{C}$. The main role of the plasma seems to be its efficiency in pre-cleaning the substrate. With the removal of the native oxide, epitaxial silicon is deposited while without the ion argon pre-cleaning, polycrystalline silicon was obtained.

The deposition of metal nitrides and oxynitrides is also one of the most important applications of low pressure plasmas for the treatment of metallic substrates and for the preparation of multilayer compounds in microelectronics [9]. Titanium nitride is one of these interesting materials since it is light and hard, anticorrosive, present a good electrical conductivity and a low friction coefficient, has the color of gold, and is efficient as a diffusion barrier [2]. The titanium nitride coatings can be produced by different technics, involving physical and/or chemical processes. The composition of $\mathrm{Ti}_{X} \mathrm{~N}_{1-\mathrm{X}}$ strongly depends on

the nitrogen vacancies in the $\delta$ crystallographic phase[10], which is drastically a function of the process used. According to the stoechiometric gap, the properties of resistivity, hardness and thermal conductivity are evolving substantially. The TiN coating can be obtained either by the CVD, or PVD (physical Vapor Deposition) or CVT (Chemical Vapor Transport), or thermodiffusional processes, each of these technics having its own limitations. They all can be assisted by an electrical discharge or not. As far as the PVD or CVT [11] technics are concerned, they require a metallic vapor, produced either via a thermal evaporation of a titanium target, or via the sputtering of the same target. There are a wide number of techniques like electron beam, ion plating, lasers, etc. which allow the production of the metallic vapor [12]. Once the metallic vapor created, it has to be transported into the reactive chamber for undergoing the reaction with nitrogen content species. In the CVD configuration, $\mathrm{TiCl}_{4}$ is decomposed, and reacts with the nitrogen content compounds to form the heavy TiN molecule. Finally, in the thermodiffusional procedure, a nitrogen content gas dissociates and adsorbs onto the surface, producing a saturation of nitrogen atoms 
onto the surface which induces the diffusion of the $\mathrm{N}$ atoms into the titanium matrix [13]. Each of these procedures can be performed using very high temperatures such as 700 to $1000^{\circ} \mathrm{C}$. The use of electrical discharge allows, for the PVD processes, to get highly dissociated reactive gas together with the titanium vapor, and hence to produce TiN at moderate temperatures such as 200 to $500^{\circ} \mathrm{C}$ [12]. Similarly, $\mathrm{TiCl}_{4}$ and $\mathrm{N}_{2}$ can be efficiently dissociated by using a glow discharge, the substrate temperature may then remain at around 200 to $500^{\circ} \mathrm{C}$ [9]. For both methods, an efficient deposition of TiN requires first the formation of titanium atoms, the transport of these atoms (convection and/or diffusion) in a reactive medium leading either to the formation of TiN in volume and deposition of the formed compound onto the surface, or to the formation of the TiN molecule by surface atom recombination reaction onto the surface [14]. For both processes, it can be interesting to polarize the substrate negatively relative to the plasma in order to grow the layer under ion bombardement. On the one hand, this lead to a cleaning of the surface during the first stage of the deposition and also to a mixing of the first TiN layers together with the substrate atoms, which improve the adhesion. On the other hand, because of the constant ion bombardment during deposition, the densification of the film is enhanced. Typically, $20 \mu \mathrm{m}$ TiN films are produced by PECVD, the hardness of which reaches 2600 Vickers [2]. However, the action of the.ions would not be used for the deposition of TiN on tortuous shaped substrates because of the non uniform resulting electric field. The effect of the plasma on the thermodiffusional technique is to increase the decomposition yields of the $\mathrm{NH}_{3}, \mathrm{~N}_{2}-\mathrm{H}_{2}, \mathrm{~N}_{2}$ ambiances via energetic electrons, leading to an increase of the nitrogen superficial concentration relative to that in the thermal procedure. In fact, atoms and radicals do not need two or more adjacent chimisorption sites, and do not present an activation energy for chemisorption as is the case for the parent molecules [12]. Moreover, the electrical discharge leads to a high hydrogen atom concentration in the gaseous medium which easily reduces the native oxide, releasing the chemisorption sites for the chemisorption of nitrogen. Once the nitrogen atoms are chemisorbed, the thermal activated diffusion process controls the growth of the layer.The enhancement of the concentration of nitrogen at the surface has been evidenced by XPS, and in the volume by Rutherford Back Scattering (table 1 and 2) $[2,15,16]$.

Table 1: O/N, N/Ti and O/Ti ratios determined by XPS on titanium treated in $\mathrm{N} 2$ or $\mathrm{NH} 3$ plasmas. $\left(\mathrm{P}=27 \mathrm{mbar}, \mathrm{D}=0.2 \mathrm{l} / \mathrm{min}, \mathrm{PI}=600 \mathrm{~W}, \mathrm{~T}=650^{\circ} \mathrm{C}\right.$, Freatment time $=1 \mathrm{H}$ )

\begin{tabular}{lccc} 
Gas & O/N & N/Ti & O/Ti \\
Plasma N2 & 37.6 & 0.06 & 2.46 \\
PlasmaNH3 & 1.93 & 0.84 & 1.62 \\
\hline
\end{tabular}

\begin{tabular}{|c|c|c|c|c|}
\hline \multicolumn{5}{|c|}{$\begin{array}{l}\text { Table 2: Incorporated nitrogen atoms into } \\
\text { the tianium matrix as a function of the power } \\
\text { discharge. }\left(10^{17} \text { atom } / \mathrm{cm} 2\right)\end{array}$} \\
\hline Power (W) & 0 & 300 & 600 & 1000 \\
\hline $\begin{array}{l}\mathrm{T}=650^{\circ} \mathrm{C} \\
\mathrm{T}=850^{\circ} \mathrm{C}\end{array}$ & 5.6 & 46 & 49 & $\begin{array}{l}14 \\
59.4\end{array}$ \\
\hline
\end{tabular}

More recently thin hard carbon layers have received wide attention because of their very attractive properties of hardness, optical transparency and band gap, thermal conductivity and/or semiconductive properties [17]. These properties are drastically a function of the crystalline quality and the hydrogen content of the films. Thus, amorphous Diamond Like Carbon (DLC), hydrogenated amorphous Diamond Like Carbon (DHLC), and Diamond thin films have been produced. The DLC and DHLC thin films have properties ranging between that of diamond and that of graphite. They are very attractive for their hardness, their transparency and their chemical stability. The diamond coatings are attractive for each of these qualities just described together with the thermal stability, very high thermal conductivity, insulating properties, and semiconductive properties when doped. The role of the plasma on the growth rate has been demonstrated without ambiguity for each of these compounds [17]. DLC and DHLC coatings will be obtained from $\mathrm{CH}_{4}+\mathrm{H}_{2}+\mathrm{Ar}$ dissociation, and with temperatures below $300^{\circ} \mathrm{C}$ $[18,19,20,21,22]$. The action of the ions is very positive, and the properties of the films are independent of the plasma frequency in experimental conditions of high impact energies of the ions impinging the substrates [23]. Consequently, under this condition, the a-C:H films properties are independent of the plasma characteristics since the low frequencies enhance processes with high thresholds, while high frequencies enhance processes of thresholds ranging between 1 to $10 \mathrm{eV}$. The intermixing of the first layers lead to very adhesive films. Diamond coatings are produced from the decomposition of a small amount of $\mathrm{CH}_{4}$ in an hydrogen plasma, together with substrate temperatures ranging from 700 to $950^{\circ} \mathrm{C}$ $[24,25,26,27]$. In contrast to polysilicon deposition, the action of ions seems to be negative. A very high concentration of hydrogen atoms allows the graphite etching, and the subsequent deposition of the metastable diamond phase. A small amount of oxygen in the discharge enhances the graphite etching rate and leads to very nicely shaped diamond crystals (figure 1) [27,28]. In contrast to the DLC films, diamond 
coatings are poorly adhesive. Cubic $\mathrm{cBN}$ is another very interesting hard material. It is thermally and chemically stable, and has good hardness and insulating properties $(5.85 \mathrm{eV}$ gap). Hexagonal hBN can be deposited from the dissociation of $\mathrm{B}_{2} \mathrm{H}_{6}$ and $\mathrm{N}_{2}$ or $\mathrm{NH}_{3}$ using an ECR (Electron Cyclotron Reactor), the deposition temperature ranging between 160 and $340^{\circ} \mathrm{C}$. An increase of the temperature together with the action of an ion flux (40 eV) transforms the hBN into $\mathrm{cBN}[8]$.
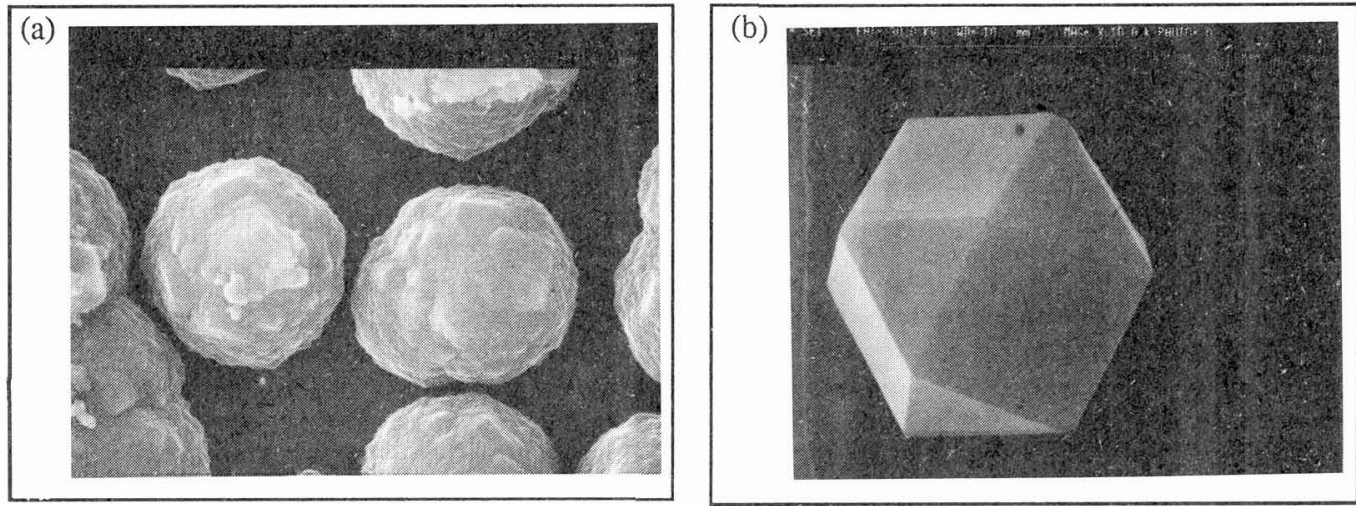

Figure 1: Influence of the chemical composition of the plasma on the shape of the diamond crystals (a) $0 \% \mathrm{O} 2$; (b) $0.5 \% \mathrm{O} 2--1 \% \mathrm{CH} 4$ in $\mathrm{H} 2$ Plasma, $400 \mathrm{~W}, 30 \mathrm{mbar}, \mathrm{T}=900^{\circ} \mathrm{C}--[27]$

\section{II- Plasma Enhanced CVD Reactors}

According to the desired coating, a large number of PECVD reactors have been developed $[17,29]$. The main electrical sources used in PECVD are the radiofrequency excitation, the microwave excitation or the hollow cathode. Each of these sources has its own advantages. Because the films properties could be completely different depending on the presence of ions bombardment or not, we will separate in two classes the reactors according to the desired effect of the ions or not, and we will try to show the specific features of each of the reactors. The way the species are activated in the gaseous medium, then transported towards the surfaces is mainly controlled by the frequency, the electrical power, the potential distribution, the electrode configuration, the substrate position, the pressure, and the hydrodynamics regime. Note that generally the lower the pressure, the higher the population of the ions relative to the neutrals.

\section{1- Reactors involving Ion effects}

Although negative ions or electrons are sometimes necessary for growing films, in most cases positive ions are needed during deposition of nitrides, oxides and silicides on silicon, stainless steel or metals. The techniques involving positive ion effects need configurations where the electrodes are internal, the substrate being maintained at the cathodic potential relatively to the plasma. These configurations are very convenient for adhesive DLC deposition $[18,19,20]$.

\section{Diode RF Reactor}

The most used design, commonly known as parallel plate reactor (figure 2) is either hot or cold walls, the plasma being created between two electrodes connected to an RF generator. In these configurations, the RF power can be coupled directly through a convertor or through a blocking capacitor [30]. The frequencies typically range between 25 and $400 \mathrm{kHz}$, or 13.56 or $27 \mathrm{MHz}$. The relation between the ground, the plasma potential and the potential of the RF electrode depends on the configuration and on the coupling. In the case of low frequency, both electrons and ions are sensitive to the RF cycles while at high frequency only the electrons respond to the variation of the electric field during a cycle. Then, in the case of a high frequency discharge and under low pressure conditions, the ions impinging on the surface are almost monoenergetic. The energy is given by the bias between the plasma potential and that of the electrode where the substrate has been placed. On the contrary, in the case where the transit time of the ions is less than the RF cycle, then a very high dispersion of the ions energy is obtained. The ions will bombard the surrounding surfaces with an widely distributed energy, the maximum on which being 
around the excitation peak bias. This diode RF reactor permits a wide range of experimental conditions depending on the desired objective. Indeed, the substrate could be placed either on the active electrode or on the grounded one. At the active electrode connected to a blocking capacitor, a cathodic sheath is formed for high frequency, and the monoenergetic ions impinging on the surface may be very fast. If the powered electrode area is smaller than the grounded parts one, a negative self bias $\left(V_{B}\right)$ relative to the ground is developed on the active electrode. The self bias depends on the ratio of the grounded surroundings and powered electrode areas $V_{B} / V_{G}=\left(A_{G} / A_{B}\right)^{n}$, with generally $n=1$, except for very low pressures where $\mathrm{n}=4[7,31]$.

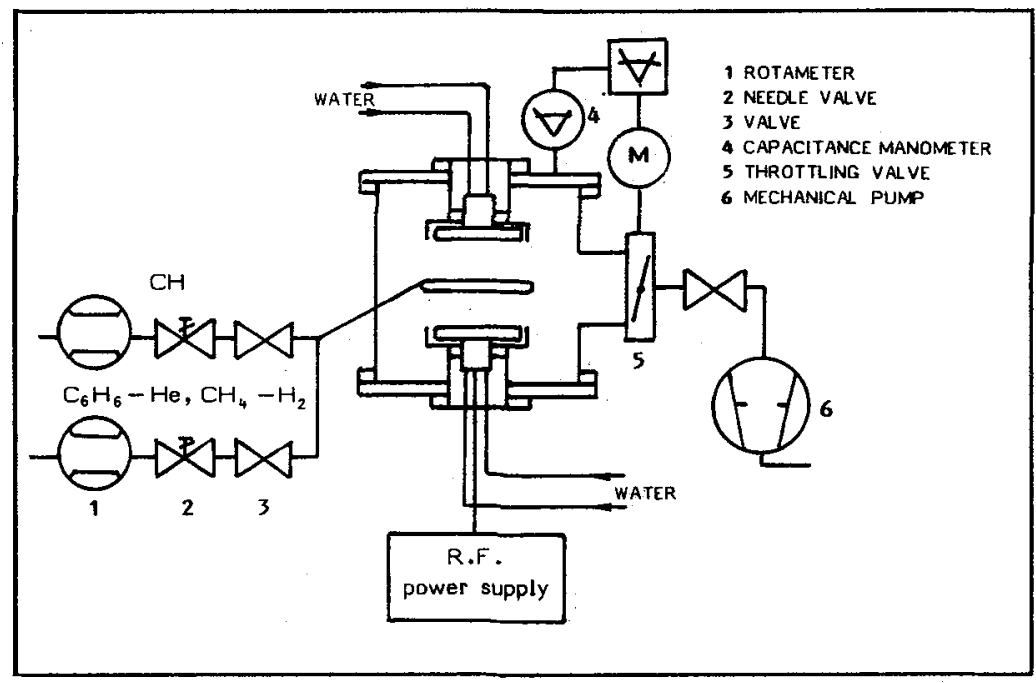

Figure 2: Outline of the RF diode reactor

\section{Pulsed RF Discharge}

In the CVD field, low temperature of the substrates must be obtained for a number of applications. Unfortunatly, using a parallel diode RF reactor would impose a very low power, and would not be compatible with high deposition rate. The use of pulsed RF reactors is very interesting since high power can be communicated through a modulated function, provoking gas dissociation together with low substrate temperatures. Figure 3 represents the evolution of the temperature as a function of the RF power for both unpulsed and pulsed RF discharges [32]. The pulsed reactor can also be used in order to vary the active species into the plasma. This is attributed to the time dependence of the concentration of the species, on the one hand formed during the active pulse and, on the other hand recombined during the non active period.

\section{Magnetron RF Discharge}

As the PECVD technique is performed at reduced pressure, the rate may remain somewhat low. Moreover, because of the surrounding surfaces (walls), defects in the films and/or in the substrate may occur. The use of magnetic field to confine the plasma has been seen to be very efficient [33]. Figure 4 [33b] shows different magnetic configurations. When magnets are placed around the reactor, the electrons take a circular motion with a Larmor radius Re. The influence of the magnetic field would be sensitive when Re is of the same magnitude as the mean free path of the electrons in the gas. For an oxygen plasma $\lambda_{e}\left(\mathrm{O}_{2}\right)=4.0310^{-2} / \mathrm{P}$ (Torr), then a magnetic field of 85 to 185 Gauss is necessary for an electron energy of around 1 to $5 \mathrm{eV}$, at 1 Torr. The magnetic field would increase ionisation since it prevents electron recombination on the wall, and then both electron density and energy are increased. Because the electron may rotate to gain energy, this technique can only be used at low pressures of around 10 mTorr. The electronic mobility in the direction normal with respect to the magnetic field will be reduced at :

$$
\mu_{\mathrm{T}}=\mu /\left[1+\left(\omega_{\mathrm{d}} / v_{\mathrm{e}}\right)^{2}\right]
$$

where $\omega_{c}=e B / m_{e}$ is the cyclotron frequency and $v_{e}$ the electron-molecule collision frequency. Typically, for 0,1 Torr and $B=100 \mathrm{G}$, the decrease of $\mu$ is around $50 \%$. Due to the reduction of the electron flux on 
the walls, the gas decomposition is enhanced, but there is a decrease of the sheath potential and of the energy of the ions impinging on the substrate. A number of designs for the distribution of the magnets around the reactor, optimizing both the energy and the density of the electrons exists.

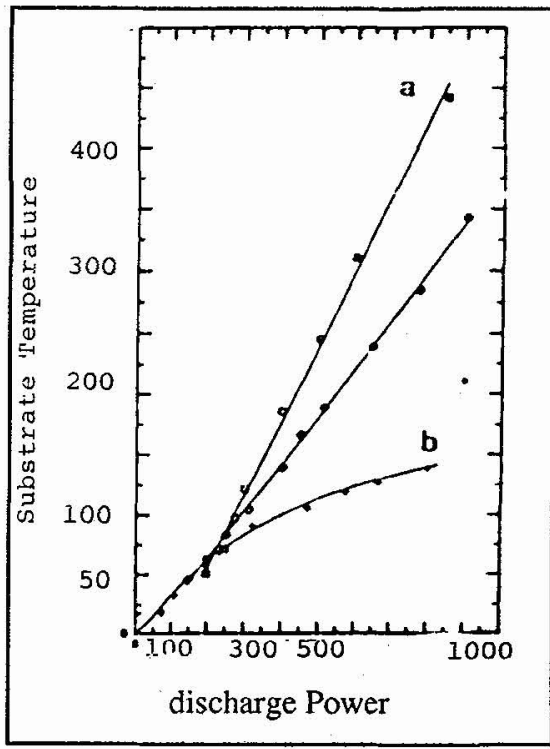

Figure 3 : Variation of the substrate temperature as the function of the power (a) Continuous, (b) pulsed RF [ 32]

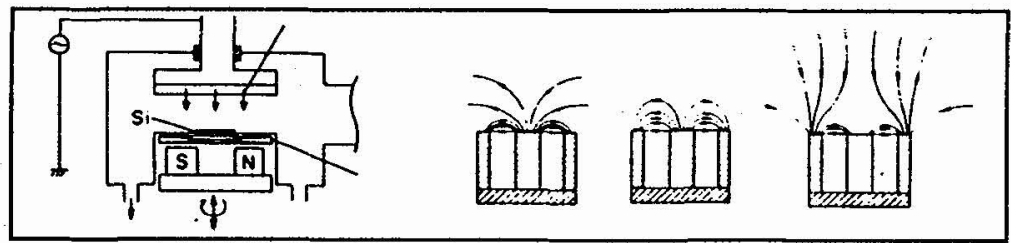

Figure 4: (a) Magnetron PECVD reactor; (b) magnetic configurations [33b]

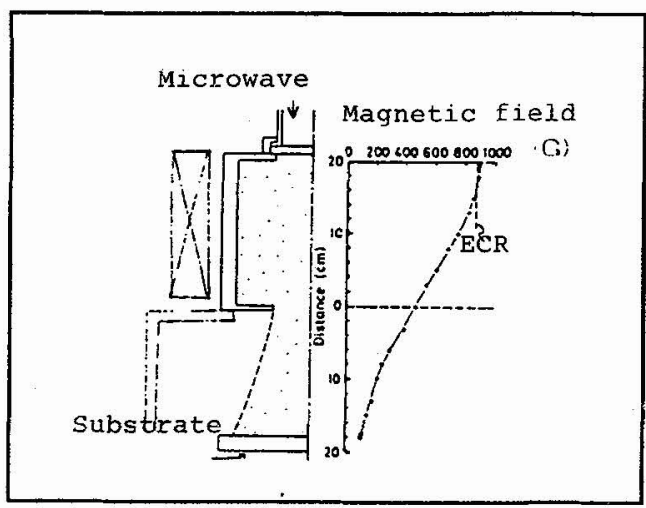

Figure 5: ECR setup 


\section{Microwave Reactors}

Microwave excitation is also widely used in PECVD techniques. In the microwave configuration, the alternative field (around $2,45 \mathrm{GHz}$ ) cannot induce a significant motion of both ions and electrons. The spatial distributions and the net balance of the different species are governed by gain and loss processes involving electronic impact, diffusion and volume recombination. For an identical power density, the electronic density is much higher in microwave than in RF discharges, even if the electron energy is lower. The net result is a more ionized, dissociated and excited medium in microwave discharges than in RF discharges. At low pressure ( $<10 \mathrm{mTorr}$ ), the ECR (Electron Cyclotron Resonance) (figure 5) [34] method has received large success. The advantage of this configuration is that it allows a continuous acceleration of the electrons (between 2 collisions) during the whole cycle. Then, high density plasmas are generated at low pressure, producing high dissociation and ionization together with a reduction of the volume nucleation, which has to be prevented in most cases. Another alternative consists in using the effect of multi-magnets on a microwave plasma, leading to the so called multipolar plasma $[35,36]$.

\section{Hollow Cathode Reactors CVT Processes}

The reactive PEPVD process or Chemical vapor transport process is an insteresting procedure to grow a number of thin films, TiN for example. The titanium source is here the hollow cathode, and very energetic argon ions extract the titanium atoms, which are then transported in a plasma consisting of a mixture of argon, nitrogen and hydrogen (figure 6) [37]. The substrates are polarized negatively relative to the plasma, and according to the distance from the substrate to the titanium hollow cathode, $\mathrm{Ti}_{2} \mathrm{~N}$ or TiN can grow. There is a correlation between the nitrogen enrichment along the flux and the nature of the deposit.

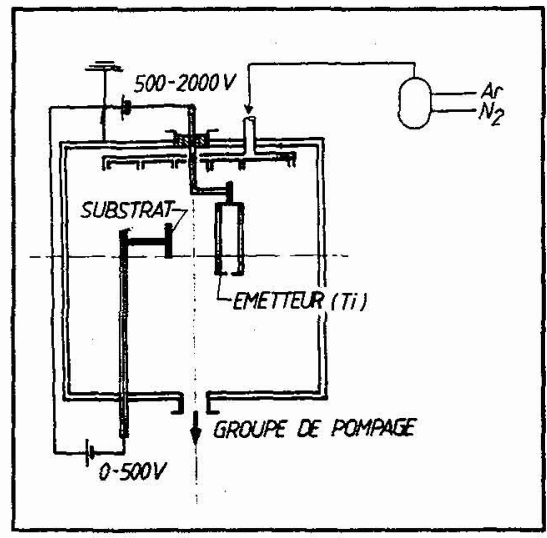

Figure 6: Titanium Hollow cathode [ 37]

\section{II.2- Reactors preventing the Ion effects}

In a number of cases, the effect of energetic ions must be prevented since it can induce some damage or lead to non uniform thin films or surface treatments. Although low pressure $\left(<10^{-1}\right.$ Torr $)$ is usually preferred in the case where ions play an important role in the process or on the resulting properties, it is not the case if the action of the ions is undesirable, since the low pressure would lead to too low deposition rates. Consequently, for all the processes which do not involve ions, the pressure will be enhanced, and electrodeless reactors or post discharges configurations will be preferred.

\section{RF or Microwave external induced plasmas}

A particular configuration of RF or microwave reactors consists in injecting the wave from external electrodes. The main advantage is to prevent any pollution from the erosion of the electrodes (figure 7) [2]. In the case where the substrate is grounded, because of the positive potential of the plasma, a cathodic sheath is formed in the vicinity of the substrate, and a capacitive component of the electric field can be seen. The ions are then accelerated and bombard the substrate almost as a monoenergetic ion beam. The dimensions of the cathodic sheath vary according to the RF or microwave power. 
In the case where the substrate is floating, a resulting steady state is the formation of a soft sheath where the ions are poorly accelerated and are then not agressive for the substrate. A number of configurations of this type have been developed for the deposition of diamond $[38,39]$, which requires a highly dissociated medium together with an absence of energetic ions [17,24]

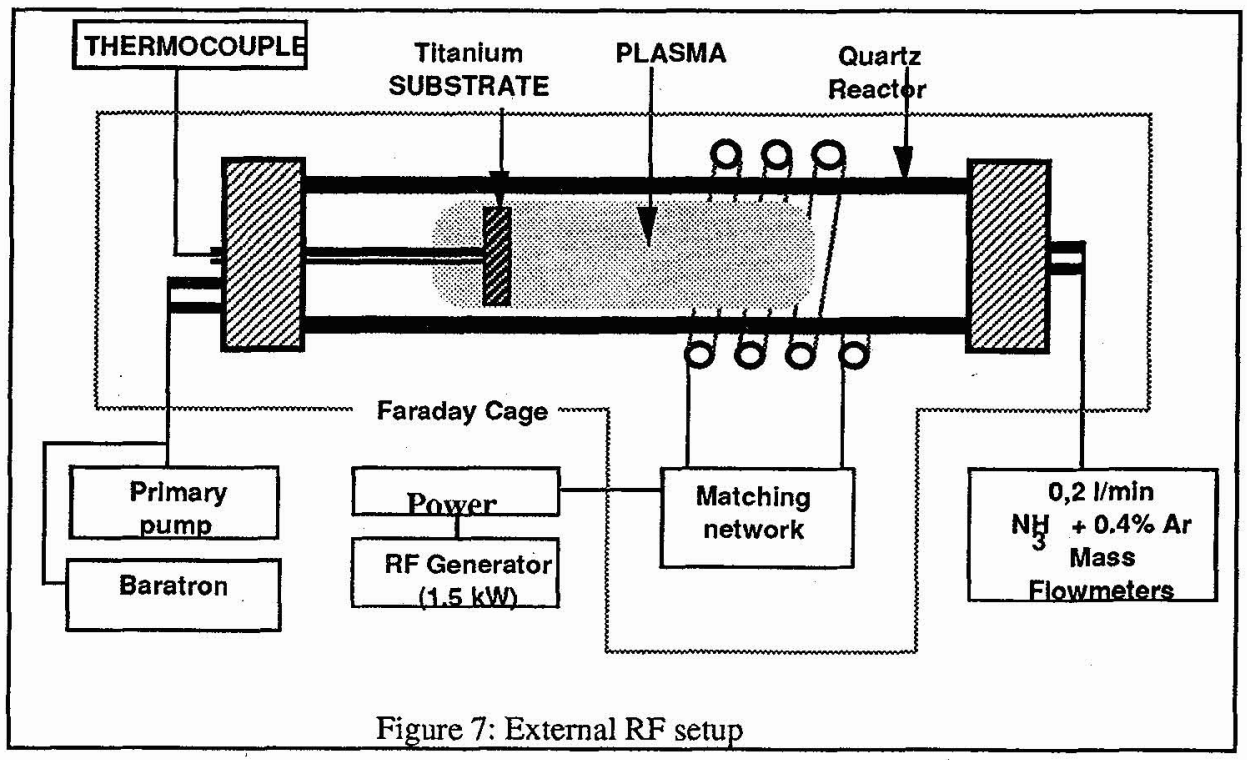

\section{Post Discharges Reactors}

The post discharge principle is the generation of a highly dissociated gas in an up stream reactor, the substrates being placed far away from this zone (figure 8) [40,41]. For a typical distance from the plasma zone, which is a function of input power, the pressure and the geometry of the reactor, there is a complete disappearance of the charged particles, and the medium is mainly composed of vibrationally excited molecules, radicals and atoms and non radiative electronic excited species. Then the substrate is not exposed to a destructive medium. In the case where the adsorption of the parent molecules on a surface present an activation barrier, there is a subsequent increase of the adsorption phenomena through these preor dissociated species. The net result is an increase of the superficial concentration of the adatoms, and an increase of the concentration gradient IN the substrate which is helpful for performing diffusion transport. Moreover, the advantage of the post discharge configuration, is that it prevents any electric field edge effect. With this technique, the effects due to the plasma (atoms and radicals concentration) and due to the substrate temperature can be separated, and then the configuration enables one to adjust independently these parameters.

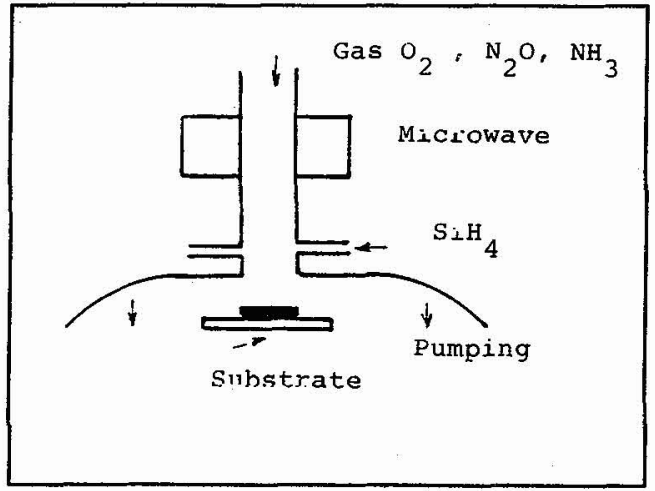

Figure 8: Post discharge Technique 


\section{III- MODELING and BASIC PROCESSES}

Experimental results and calculations $[42,43,44,45]$ have demonstrated that fluid flow and transport phenomena strongly influence the deposition characteristics (uniformity, growth rate) and surface treatment. The modelling of thermally activated CVD reactors is already very difficult, the addition of the plasma processes still increase the complexity of the modeling.

Beyond the phenomena induced during any deposition in a thermal CVD reactor, in PECVD, we must consider the processes related to the nature of the plasma. While in thermal CVD, the main chemical reactions producing the active species take place at the surface, in PECVD most of them are taking place in the gaseous phase. Futhermore, in the case where the ions constitute the species responsible for the deposition, the processes occuring in the cathodic sheath must be taken into account. Because of the difference in the active species impinging on the surface, the surface chemical reactions are somewhat different and the mass transfer efficiency should vary. As far as the heat transfer is concerned, consisting mainly in a transfer from the hot surface to the gaseous molecules in thermal CVD, in PECVD, it results from a number of processes. Besides an external heating, the surface receives heat from the processes of accomodation of vibrational and rotational energies, of accomodation of atoms and radical recombination reactions $[46,47,48,49]$, of ion momentum transfer and of adsorption, the heat losses at low pressure being mainly due to radiation. All these processes may in turn influence the hydrodynamics and the characteristics of the plasma in the vicinity of the substrate. The modeling of both the main reactive flowing plasma and the plasma/surface interface is rather difficult because of the number of phenomena involved. A systematic classification of the phenomena as a function of their magnitude needs to be done, and the coupling between the main phenomena must be taken into account.

\section{III.1- Main Reactive Flowing Plasma}

The so main reactive flowing plasma represents the gaseous phase flowing where the chemistry under non equilibrium situation is taking place. Typically, the composition of such a medium is electrons, the energy of which ranges between 1 to $20 \mathrm{eV}$, ions, molecules in a variety of excited states (electronically excited, ground electronic state - vibrationally and/or rotationally excited states), radicals and atoms. The modeling of the flowing plasma used in chemical vapor deposition techniques needs to identify each of the processes of creation and loss of these species and the processes by which they gain or lose energy. It also needs the knowledge of the hydrodynamic regime.

Generally, the hydrodynamics of the flow (laminar or turbulent) far from the substrate can be well described by the 2D or 3D Navier Stockes motion equation.

For the modeling of the electron distributions both in energy and in density, Boeuf [50] has developed hybrid models (Fluid-Monte Carlo models) taking into account four mechanisms representing the electron creation and the way they gain energy in an RF discharge. The first ones are emitted from the cathode, the second ones named "wave-riding" electrons gain energy under the RF cycles into the sheath. The third ones exist at very low pressure such that the electron mean free path is of the same magnitude than as the discharge characteristic length, in which case the electrons have an increased probability of impinging the sheath. Finally, the fourth ones are the electrons in volume which are accelerated via the plasma electric field.

The energy transfers resulting from electron-molecule collisions and from molecule-molecule non reactive collisions lead to a highly non equilibrium states of the low pressure plasma. Actually, the inelastic collisions induce internal energy tranfers such as V-V, V-R and V-T $[51,52,53]$. Each of these processes is characterized by a cross section, the values of which are not always available in the literature. In particular, very little is known of the electron-molecule collision cross section leading to dissociation, ionization and excitation of the particles, except for the rare gases. Moreover, for the production of a specified state, different ways are possible which must be taken into consideration. The conventional reactions involving molecules, atoms and radicals obviously occur in such a medium. However, although they are still strongly dependant on the gas temperature, they will be enhanced by vibrational or rotational excitation according to their exothermicity [54] as demonstrated by Polanyi and Lee [55].

As a result, the modeling of a reactive flowing plasma is very complex. Because of the complexity of the processes and the high number of them, choices are often done in order to get an approximated satisfactory modelling underlying one or two specified phenonema. 
In order to give an illustration, we propose now to deal with two examples which have been treated by the authors. In the first one, the purpose was to deposit amorphous silicon onto silicon wafers by means of the decomposition of silane in a tubular reactor at a total pressure of 0.2 Torr, including $10^{-2}$ Torr of SiH4. The discharge was supplied by external electrodes connected to an RF generator $[56,57,58,59]$. The second example concerns the thermodiffusional nitridation of titanium in a tubular low pressure ( 5 mbar) ammonia plasma, the titanium substrates being placed down stream, in the stagnation point configuration. The discharge was also created by an external RF electrode. For both cases, to a first approximation, in the flowing plasma, the mono-dimensional transport equations were used for each species. For both cases, a kinetic mechanism has been postulated, and the electron-molecule collisional reaction rate constant, not available in the literature, have been determined experimentally. Catherine et al $[60,61]$ has thus solved analytically the decomposition of silane by assuming a global first order reaction rate relative to the silane for the $\mathrm{SiH} 4$ decomposition reaction. While Gicquel et al $[62,63]$ have calculated numerically the composition of the flowing plasma along an $\mathrm{NH}_{3} \mathrm{RF}$ plasma tubular reactor, and determined experimentally an elementary electron- $\mathrm{NH}_{3}$ collisional rate constant. These formulations could give interesting results since in both cases, the consumption of the active species by the substrates along the flowing plasma was negligible compared to the homogeneous chemical reaction production.

In first approximation, a one dimensional continuity equation for a species $M$ is written:

$$
u_{m} d\left(N_{M}\right) / d z-D_{M}\left[\left(d^{2} N_{M}\right) / d z^{2}\right]=R_{M}
$$

where $u_{m}$ is the mean flow velocity, $D_{M}$ the diffusion coefficient of the $M$ species, $z$ the reactor axis, and $\mathrm{RM}$ the net production rate of the $\mathrm{M}$ species.

For the silane decomposition, the decomposition was supposed to result from the following global reaction:

$$
\mathrm{SiH}_{4}+\mathrm{e}^{-}-\mathrm{k}_{-}>\mathrm{SiH}_{n}+\mathrm{H}(1-n)+\mathrm{e}^{-}
$$

$\mathrm{SiH}_{4}$ decrease and $\mathrm{SiH}_{\mathrm{n}}$ radicals profiles were then described by:

$$
\text { for } \mathrm{SiH}_{4} \text { : } \quad \mathrm{N}_{\mathrm{m}}=\mathrm{N}_{0} \exp (-\mu z)
$$

with $\mu=-\left(\mathrm{u}_{\mathrm{m}} / 2 \mathrm{D}_{\mathrm{M}}\right)+\left[\left(\mathrm{u}_{\mathrm{m}}^{2} /\left(4 \mathrm{D}_{M}^{2}\right)\right)+\left(\mathrm{k}_{1} / \mathrm{D}_{M}\right)\right]^{1 / 2}=1 / \mathrm{R}\left[-\left(\mathrm{P}_{\mathrm{e}} / 2\right)+\left(\left(\mathrm{P}_{\mathrm{e}}^{2 / 4}\right)+\mathrm{a}\right)^{1 / 2}\right]$, where $\mathrm{k}_{1}=\mathrm{n}_{\mathrm{e}^{\mathrm{k}}}$ ( $\mathrm{n}_{\mathrm{e}}$ electron density and $\mathrm{k}$ electron-silane collisional rate constant $), \mathrm{P}_{\mathrm{e}}=\left(\mathrm{u}_{\mathrm{m}} \mathrm{R} / \mathrm{DM}\right)$ is the Peclet mass number which measures the relative importance of forced convection and diffusion, and $a=\left(k_{1} R^{2} / D_{M}\right)$, the Damkhöler number, which measures the importance of the homogeneous reaction relative to diffusion, $\mathrm{N}_{0}$ represents the initial silane concentration, and $\mathrm{R}$ the radius of the reactor.

\section{for the SiHn Radicals:}

$$
N_{R}=A\{\exp (-\mu z)-\exp (-\lambda z)\}
$$

with $\lambda\left(\mathrm{cm}^{-1}\right)=-\left(\mathrm{u}_{\mathrm{m}} / 2 \mathrm{DM}\right)+\left\{\left(\mathrm{u}_{\mathrm{m}}^{2} /\left(4 \mathrm{DM}^{2}\right)\right)+(2.405 / \mathrm{R})^{2}\right\}^{1 / 2}=1 / \mathrm{R}\left[-\left(\mathrm{P}_{\mathrm{e}} / 2\right)+\left(\left(\mathrm{P}_{\mathrm{e}}^{2 / 4}\right)+5.78\right)^{1 / 2}\right]$, and $A\left(\mathrm{~cm}^{-3}\right)=\mathrm{k}_{1} \mathrm{~N}_{0} /\left\{-\left(\mathrm{D}_{\mathrm{M}^{2}} \mu^{2}\right)+\mathrm{u}_{\mathrm{m}} \mu+5.78\left(\mathrm{D}_{\mathrm{M}} / \mathrm{R}^{2}\right)\right\}$

In the case where the diffusion is slow relative to the homogeneous chemical reaction $(a>5.78)$, thus, the axial depletion is governed by $\lambda$. For the inverse situation, the axial depletion is controlled by $\mu$.

For the deposition step of amorphous silicon, since an axial distribution existed, the radial flux of radicals $\Gamma_{r}=-D_{R} \partial N_{R} / \partial r \cong b\left(D_{R} / R\right) N_{R}$ (b is a dimensionless global coefficient) which controls the mass flow towards the substrate transport depended on this axial distribution. As the surface reaction was very fast, the radial flux was reflecting exactly the $\mathrm{NR}_{R}$ axial profile. The experimental results have confirmed this assumption, and that the global process was controlled by the reaction in the flowing plasma. This has justified, a postiori, that we could use such a simple model. 
The global reaction rate constant $\mathrm{k}_{1}$ was deduced from the comparison of the amorphous silicon deposition rate measured experimentally and that calculated from the model (figure 9). $\mathrm{k}_{1}$ was varying from $12 \pm 1 \mathrm{~s}^{-1}$ to $93 \pm 8 \mathrm{~s}^{-1}$ as the power was varying from 10 to $200 \mathrm{~W}$, which are of the same magnitude of the values found in the literature $\left(\mathrm{k}_{1}=10-70 \mathrm{~s}^{-1}\right)$ [61].
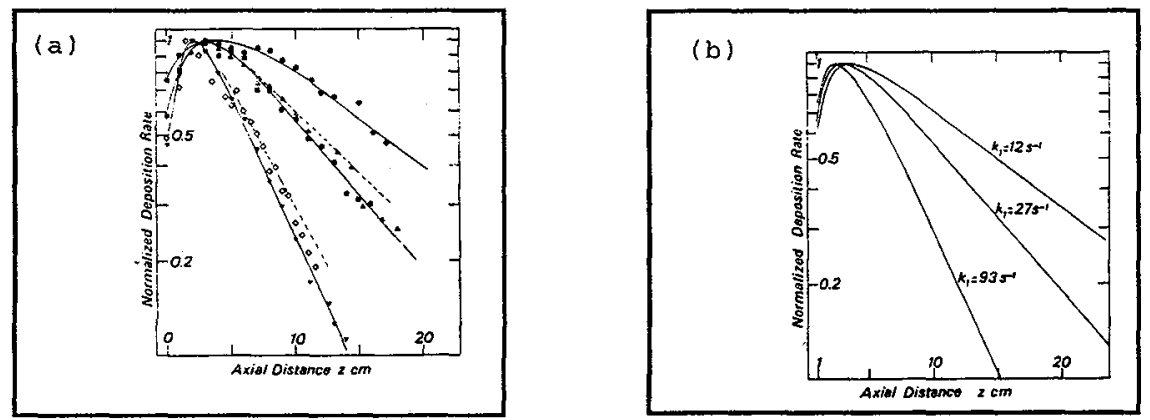

Figure 9: Amorphous silicon deposition from the silane decomposition - Normalized deposition rate vs axial distances for different RF powers.(a) experimental (b)Theoretical 0.10w; $\triangle .20 \mathrm{w}: \mathrm{n}, 50 \mathrm{~W}$ $\square, 100 \mathrm{w}: \mathrm{\nabla}, 200 \mathrm{w}$

For the modelling of the composition of the $\mathrm{NH}_{3}$ flowing plasma, a $4.5 \mathrm{~cm}$ diameter tubular reactor has been used. The main reactive flow was localized inside a $3 \mathrm{~cm}$ diameter tube, due to the confinement of the flowing plasma produced by the magnetic field and the presence of a capacitive electric field generated between the RF inductive copper rings and the $3 \mathrm{~cm}$ diameter titanium substrate (figure 7). A reaction mechanism (table 3 ) has been formulated $[62,64]$ to describe the plasma dissociation of ammonia. In the kinetic model, the influence of the rovibrational excitation on the heavy particles reaction rate constants were not taken into account, and the constants were then varying according to the Arrhenius law. We also have assumed that each $\mathrm{N}-\mathrm{H}$ and $\mathrm{H}-\mathrm{H}$ bond cracking due to electron collisions have the same magnitude for the corresponding rate constant, which was unknown. The determination of a vibrorotational $\mathrm{NH}_{3}$ molecule electron collisional reaction rate constant has been possible for a given set of experimental conditions using laser absorption spectroscopy. Actually, this allowed us to measure the axial $\mathrm{NH}$ partial pressure distribution. The profile of the $\mathrm{NH}$ partial pressure along the reactor showed a maximum at around the middle of the reactor (figure 10). This expresses, after a certain distance from the RF excitation source, the net balance between on one hand the radical production, and on the other hand, the radical destruction. We assumed that the situation was the same for $\mathrm{NH}_{2}$. This relationship was verified. A relatively simple expression for the relationship between the $\mathrm{NH}$ partial pressure and the $\mathrm{NH}_{3}$ partial pressure has then been obtained, in equalizing the production rate and the loss rate for both NH and $\mathrm{NH}_{2}$. In order to get an estimate for the electron collisional dissociation rate constant, keeping constant the electron temperature and electron density product, measurements of partial pressure of $\mathrm{NH}$ and rotational temperature have been performed as a function of total pressure in the bulk plasma region, where the NH partial pressure gradient was equal to zero. The corresponding plot gave rise to a $\mathrm{k} \mathrm{x} \mathrm{n}_{\mathrm{e}}$ electron collisional reaction rate constant of $1 \pm 0.5 \mathrm{~s}^{-1}$ (at $1500 \mathrm{~K}$ and at a plasma power of $700 \mathrm{~W}$ ).

Assuming then that in the flowing plasma, far from the titanium substrate, the transport by convection is high compared with the transport by diffusion, the numerical resolution was performed using the Chemkin code from Sandia Laboratory, taking into account an axial gas temperature profile along the reactor. The temperatures of the profiles were assumed to be equal to the rotational temperatures which were measured on the $\mathrm{N}_{2} \mathrm{C}^{3} \pi_{\mathrm{u}}$ electronic excited state.

The results of the model for a typical set of conditions, is illustrated in figure 11. Although the assumptions above are highly simplified, the results (model (for $\mathrm{kn}_{\mathrm{e}}=0.54 \mathrm{~s}^{-1}$ ) and experiments) were in a relatively good agreement for times well before the flowing plasma is influenced by the titanium surface. Of course, the surface induced kinetics were not included in the reaction set, and a comparison should only be made for times less than $45 \mathrm{~ms}$. Moreover, the decomposition rates (X) obtained from the model and from experiments (in absence of the catalytic titanium) were also in a relatively good agreement. 


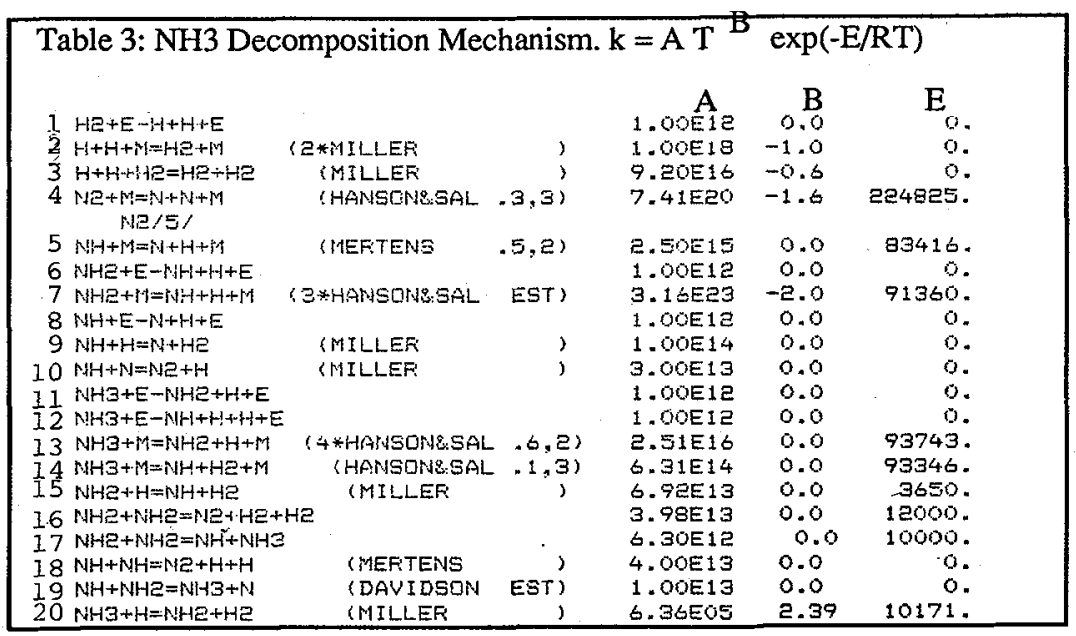

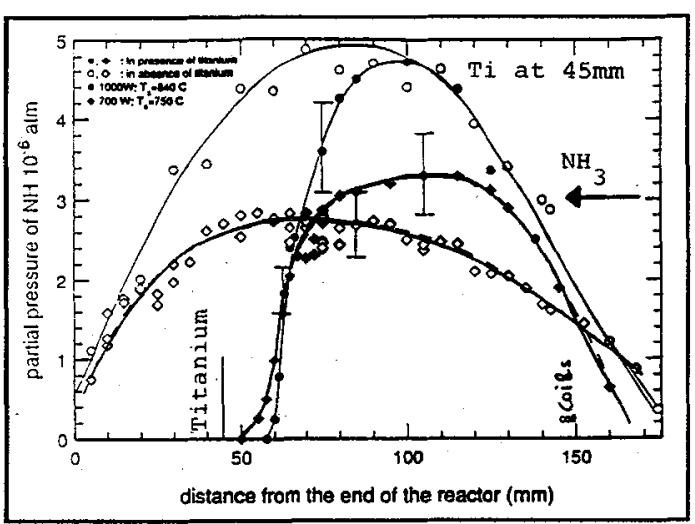

Figure 10: NH partial pressureProfiles along the reactor, in presence and in absence of the titanium

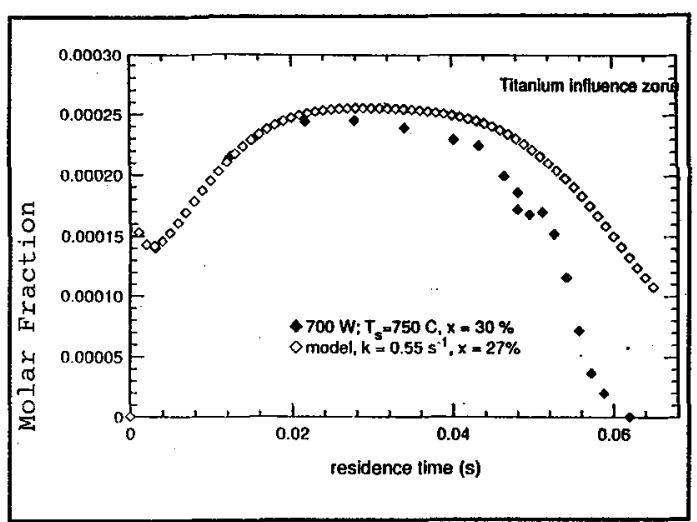

Figure 11: NH Partial pressure profilescomparison between experience and modeling

Therefore as a result, we have been able to determine an approximate value for the electron collisional rate constant for the $\mathrm{NH}_{\mathrm{x}}+\mathrm{e}-->\mathrm{NH}_{\mathrm{x}-\mathrm{y}}+\mathrm{e}+\mathrm{H}_{\mathrm{y}}(\mathrm{x}=1$ to $3, \mathrm{y}=1$ or 2$)$ reaction and to model the dissociation kinetics giving the main reactive species distribution, even these which were not accessible through experimental measurements .

\section{III.2 Plasma / surface Interface}

The plasma / surface interface modeling will strongly depend on the main deposition driving species. As a matter of fact, one can assist either processes dominated by the ionized species and the electrons in volume and / or at the surface, or processes dominated by the dissociated species in volume and / or at the surface. Moreover, it also will depend on the importance of the transport by convection relative to that by diffusion towards the substrate. Thus, with small Peclet numbers, the active species will be transported under a diffusional flux, and the deposition rate will be influenced by the spatial distribution in the reactor. On the contrary, in the case of high Peclet numbers, the deposition rate will not be influenced by the species distribution into the reactor. The only limitation could be either the homogeneous reactions rates depending on the plasma parameters, or the surface reactions rates. 
In the case where the deposition is carried out by the ions, the reactive species distribution in the reactor will not influence the deposition rate, since the active species will only be created in the cathodic sheath. The deposition rate will strongly depend on the characteristics of the sheath, responsible for the ion energy and density. This has been underlined by Catherine et al for the deposition of diamond like carbon films, by modifying the RF frequency without observing any difference on the deposition rate.

\section{Conclusion}

This key note lecture has been focused on the use of plasmas for chemical vapor deposition. The advantages of PECVD have been underlined: beside the net decrease in the substrate temperature, it allows the introduction of doping species during the treatment, the creation of new species, and the possibility of adding the action of energetic ions. We have reviewed the main basic reactors together with their specific characteristics. A compromise between the dissociating power of the plasma and the temperature, or between the ion density and the uniformity of the deposit may be sometimes necessary. The choice of a good configuration and the adequate balance of the main parameters leading to the control of the process may result from a deep understanding of the basic phenomena. A detailed analysis of the phenomena provides sometimes evidence for the most important ones. The modeling of these main processes may then be realized leading to simplified models. An illustration has been proposed where the one dimensional continuity equation was used. Kinetics models have been postulated involving a number of electron-molecule collisional reactions, the rate constants of which were not available in the literature. Experiments were then designed in order to extract these rate constants, representing either global or elementary reactions. The resulting models allowed one to get information on species not accessible experimentally. More sophisticated models, including 2D or 3D hydrodynamics, electron characteristics, molecule-molecule and molecule-surface energy transfer and surface chemistry, are needed to describe the plasma/surface interface and the resulting mass transfer from the plasma towards the substrate.

\section{Acknowledgments:}

The authors gratefully acknowledge Professor A. Vignes, from the Ecole Nationale Supérieure de Chimie de Paris, for hepful discussions.

\section{References:}

/1/ M. Konuma, , O. Matsumoto, Y. Konzaki, J. of the less common metals, 75 (1980) 1-5

12) A. Gicquel, N. Laidani, P. Saillard, J. Amouroux, Pure \& Appl. Chem. vol 62, $n^{\circ} 9(1989)$ 1743-1750

13/ J. Perrin, Thèse d'Etat, Univ. Paris VII (1983)

14/ B. Coll, P. Jacquot, Surface and Coatings Technology, 36, 867.878 (1988)

15/ G. Turban, Y. Catherine, B. Grolleau, Thin Solid Films, 60 (1979) 147-155

16/ B. E. Chin, E. P. Van de Ven, Solid State Technolo. (1988) 119

171 T. J. Donahue, R. Reif, J. Electrochemical Society, 139 (1986) 169, R. Reif, "PECVCD for Microelectronics"

18/ Y. Catherine, Le Vide et les Couches Minces, Supplếment au n²46 (1989) 172179

191 P. Bradu, D. Degout, A. Gicquel, A. Ricard, Revue Scientif. et Techn. de la Defense, (1990) 71-86

/10/ J. P. Bars, E. Etcheessahar, J. Deguigne, J. of the Less Common Metals, 52, 51-76 (1977)

/11/ W. Piekarczyk, R. Messier, R. Roy, C. Engdahl, J. Crystal Growth 106 (1990) 279-293

112/ P. Jacquot, Thèse de Docteur INPL (1985)

/13/ N. Laidani, J. Perrière, D. Lincot, A. Gicquel, J. Amouroux, Appl. Surface Sciences, 36 (1989) 520 -

/14/ V.V. Quichaud, D. Dézert, J. Aubreton, D. Degout, A. Catherinot, Laser Chem. 10 (1989) 93

/15/ P. Saillard, A. Gicquel, J. Amouroux, N. Moncroffre, J. Tousset, Surface and Interface Anal., vol 16 (1990) 530-

116/ P. Saillard, A. Gicquel, J. Amouroux, Surface and Coating Technol. (1991) to be published

117/ Y. Catherine, A. Gicquel, "Les dépôts de Carbone Dur: Films Adamantins et Diamant" CIP (1991)

/18/ Y. Catherine, P. Couderc, Thin Solid Films, 144 (1986) 265-280

119/ Y. Catherine, G. Turban, B. Grolleau, Plasma Chem. and Plasma Proc., vol 2, $\mathrm{n}^{\circ} 1$ (1982) 81-93

120/ P. Couderc, Y. Catherine, Thin Solid Films, 146 (1987) 93-107

/21/ Y. Catherine, A. Pastol, E-MRS, vol XVII, (1987) 145-151

1221 Y. Catherine, A. Pastol, L. Athouel, C. Fourrier, IEEE Trans. on Plasma Science, vol $18 n^{\circ} 6(1990) 923-$

123/ Y. Catherine, Mat. Science Forum, vol 52 \& 53 (1989) 175-196

124/ A. Gicquel, Technical Report "Synthèse des Films Minces de Diamant" SGDN, (1989)

125/ B. V. Spitsyn, L.L. Bouillov, B. V. Dejarguin, J. of Crystal Growth (1981) 219-226

126/ K. Kobashi, K. Nishimura, Y. Kawate, T. Horiuchi, Phys. Rev. B, Cond. Matter, vol 38, nº (1988) 4067

$127 /$ A. Gicquel, C. Héau, E. Anger, D. Fabre, F. Cansell, J. P. Petitet, Proc. ADC'91 (1991) to be published

1281 A. Gicquel, C. Héau, E. Anger, D. Fabre, F. Cansell, J. P. Petitet, Proc. Diamond Film 91 (1991)

129/ Y. Catherine, Course on "Preparation Techniques for Diamond Like Carbon", Pascoli, Italy (1990) 
130/ K. Kohler, J. W. Coburn, D. E. Horne, E. Kay, J. H. Keller, J. Appl. Phys. 57, (1985) 59

131/ D. Flamm, Microelectronics and Surface Technol. course CVD and PECVD France Feb. (1990)

132/ F. El Hossary, D. J. Fabian, A. P. Webb, IPAT 87, $6^{\text {th }}$ Int. Conf. on Ion and Plasma Assisted Techniques(1987)

133/ T. M. Yuzuhira, W. E. Mlynko, D. W. Hess, J. Vac. Sci. Technol. A 3, (1985) 2135

133b/ B. Window, N. Savvides, J. Vac. Sci. Technol. A4, (1986) 196

/34/ M. R. Wertheimer, M. Moisan, J. Vac. Sci. Technol. A 3, (1985) 2643

135/ R. R. Burke, C. Pomot, Solid State Technology (1988) 67

136/ Y. Arnal, A. Durander, J. Pelletier, M. Pichot, L. Vallier, CIPG Antibes, France (1987) 73

137/ A. Ricard, J. Oseguera, H. Michel, M. Gantois, PSE Conf., Garmisch (1988)

138/ C. P. Chang, D. L. Flamm, D. E. Ibbottson, J. A. Mucha, J. Appl. Phys. 63 (5) (1988)

139/ P. K. Bachmann, R. Weimer, R. Messier, Proc. Diamond of the Diamond Techn. Initiative Symposium T3 (1988)

140/ A. Ricard, G. Henrion, H. Michel, M. Gantois, Pure and Appl. Chem. 60 (1988) 747

141/ R. L. Jackson, J. E. Spencer, J. L. Mc Guire, A. M. Hoff, Solid State Technol. 107 (1987)

/42/' C. R. Kleijn, C. J. Hoogendoorn, Chem. Engineer., vol46, ${ }^{\circ} 1$ (1991) 321-334

143/ S. Rhee, J. Szekely, $3^{\text {rd }}$ Intern. Modeling of Casting and Welding, Sta Barbara (1986) 52-78

144/ M. Gantois, D.Ablitzer, J. L. Marchand, H. Michel, A. Ricard, $6^{\text {th }}$ Cong on Heat Treatment of Mat.,(1988)

145/ Jensen, Conference on the modelization, San Franscico (1988)

146/ B. Halpern etD. E. Rosner, J. Chem. Soc. Faraday Trans. 160 (1978) 1883-1912

147/ A. Gicquel, Thèse d'Etat, Univ. Paris VI (1987)

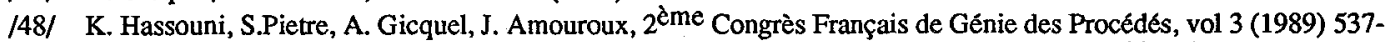

149/ K. Hassouni, U. Uktu, S. Cavvadias, A. Gicquel, J. Amouroux, Proc. ISPC 10, IUPAC, (1991) Germany

150/ J. P. Boeuf, Phys. and Appl. of Pseudosparks, Nato, ASI series, Ed Gundersen aShaefer, Plenum Press (1990)

151/ M. Capitelli, Topics in Current Chemistry, $n^{\circ} 90$ (1980) 60-109

152/ M. Capitelli, C. Gorse, A. Ricard, Topics in Current Physics, vol 39 (1986)

153/ S. de Benedictis, A. Gicquel, F. Cramarossa, ISPC 8, IUPAC, vol 1 (1987) 637

154/ A. Gicquel, P. Saillerd, N. Laidani, J. Amouroux Rev. Phys. Appl., 24 (1989) 285

155/ J. C Polanyi, Acc. Chem. Res. 5 (1972) 161

156/ G. Turban, Pure \& Appl. Chem., vol 56, n², (1984) 215-230

157/ G. Turban, Y. Catherine, B. Grolleau, Thin Solid Films, 77 (1981) 287-300

158/ G. Turban, Y. Catherine, B. Grolleau, Plasma Chem. and Plasma Proc., vol 2, n 1 (1982) 61-79

159/ J. Perrin, J. P. M. Schmitt, G. de Rosny, B. Drevillon, J. Hut, A. Lloret, Chem. Phys., 73, (1982) 383

/60/ G. Turban, Y. Catherine, Thin Solid Films, 48 (1978) 57-65

/61/ G. Turban, Y. Catherine, B. Grolleau, Thin Solid Films, 60 (1979) 147-155

162/ A. Gicquel, M. Cappelli, A. Chang, R. Hanson, ISPC 9, IUPAC vol III (1989) 1613-1618

163/ A. Gicquel, P. Saillard, S. Pietre, M. Cappelli, J. Amouroux, Proc. ISPC 10, IUPAC, (1991) Germany

/64/ D. Davidson, private communication 